\title{
FATORES DE RISCO NÄO HABITUAIS PARA METÁSTASE LINFONODAL NO CÂNCER DO COLO DO ÚTERO
}

\author{
José Humberto tavares Guerreiro fregnani*, fernando Augusto Soares, Pablo Roberto Novik, Ademar lopes, Maria do Rosário Dias de \\ Oliveira Latorre \\ Trabalho realizado no Centro de Tratamento e Pesquisa Hospital do Câncer A. C. Camargo. Trabalho financiado pela Fundação de Amparo à Pesquisa do Estado
} de São Paulo

\author{
* Correspondência \\ Departamento de Morfologia \\ (Faculdade de Ciências Médicas \\ da Santa Casa de São Paulo) \\ Rua Dr. Rua Cesário Motta \\ Júnior, 61 \\ Cep: 01221-020 - São Paulo/SP \\ Tel. / Fax: 2176-7000 \\ ramal 5509 \\ mdfregnani@terra.com.br
}

\begin{abstract}
RESUMO
Oвjetivo. Verificar as variáveis associadas às metástases nos linfonodos pélvicos em pacientes com carcinoma do colo do útero nos estádios IB e $\|$.

Métodos. Estudaram-se 289 pacientes admitidas no Departamento de Ginecologia do Centro de Tratamento e Pesquisa Hospital do Câncer A. C. Camargo com carcinoma invasor do colo do útero (B e IIA) no período de 1980 a 1999 e que foram submetidas à cirurgia radical. A coleta de dados foi realizada a partir dos prontuários e da revisão de cortes histológicos dos colos de útero e dos linfonodos, sendo registrados dados sociodemográficos (idade ao diagnóstico, cor da pele), clínicos (estádio da doença, taxa de hemoglobina pré-operatória e índice de massa corpórea) e histopatológicos (tipo histológico, grau histológico, índice mitótico, embolização em capilares linfáticos e/ou sangüíneos, invasão perineural, profundidade de invasão do tumor no estroma cervical, intensidade da reação inflamatória no colo do útero, necrose tumoral, tamanho do tumor, invasão dos ligamentos cervicais laterais e do corpo do útero e status linfonodal). A associação entre a presença de metástase linfonodal e as diversas variáveis foi avaliada pelo teste de Qui-quadrado, pelo teste exato de Fisher e pela regressão logística múltipla.
\end{abstract}

Resultados. Encontrou-se metástase nos linfonodos pélvicos em 65 pacientes (22,5\%). Foram identificados os seguintes fatores de risco para metástase linfonodal na análise multivariada: taxa de hemoglobina < I0,0 g\% (OR=3,6; IC95\%: I,210,7), invasão tumoral do terço médio (OR=3,3; IC95\%: I, I- I0,7) e profundo do colo do útero $(O R=5,4$; IC95\%: I,717,3), reação inflamatória ausente ou de intensidade leve no colo do útero (OR=2,4; IC95\%: I, I-5,2) e carcinoma epidermóide queratinizante $(\mathrm{OR}=3,3 ; \mathrm{IC} 95 \%: 1,4-7,6)$.

Conclusöes. Este estudo identificou quatro fatores de risco para metástase nos linfonodos pélvicos em pacientes com carcinoma do colo do útero nos estádios IB e IIA. Destes, três não são habitualmente descritos na literatura como variáveis preditoras de metástase linfonodal (anemia pré-operatória, reação inflamatória ausente ou de intensidade leve e carcinoma epidermóide queratinizante).

Unitermos: Neoplasia do colo do útero. Metástase linfática. Linfonodos. Anemia. Inflamação.

\section{INTRODUÇÃO}

Nos países desenvolvidos, o carcinoma do colo do útero representa $4,2 \%$ de todos os tumores em mulheres e é o sexto tipo mais freqüente de câncer na população. Nos países em desenvolvimento é o primeiro ou o segundo tipo de câncer mais comum no sexo feminino, correspondendo a $15 \%$ dos tumores!.

O tratamento do carcinoma do colo do útero é ditado pelo estadiamento clínico estabelecido pela Federação Internacional de Ginecologia e Obstetrícia². Os estádios iniciais IB e IIA podem ser tratados por histerectomia radical classe III de Piver-Rutledge ${ }^{3}$ associada à linfonodectomia pélvica ou através da radioterapia (teleterapia pélvica associada à braquiterapia vaginal). Nos estádios mais avançados (IIB até IVB), a radioterapia apresenta melhores resultados que a cirurgia ${ }^{4}$.

Cerca de 20\% das pacientes portadoras de carcinoma do colo do útero em estádios iniciais (IB e $\| A$ ) e que são adequadamente tratadas por cirurgia radical desenvolvem recorrência da doença ${ }^{5}$. Em 90,0\% das vezes, ela acontece em um prazo de dois anos 6 . A presença de metástases nos linfonodos pélvicos é seguramente 0 principal fator de risco para recorrência do carcinoma do colo do útero, observando-se significativa redução da sobrevida das pacientes que as possuem?.

Os fatores de risco para metástase nos linfonodos no carcinoma do colo do útero habitualmente descritos na literatura são a profundidade de invasão do tumor no estroma cervical, o acometimento do ligamento cervical lateral ("paramétrios"), a embolização capilar linfática e/ou sanguínea, o grau histológico, o tamanho do tumor e o estadiamento clínico-9.

Este estudo tem por objetivo avaliar os fatores de risco para a metástase nos linfonodos pélvicos em pacientes com carcinoma do colo do útero tratadas inicialmente através de cirurgia radical, considerando-se na análise não apenas as variáveis que usualmente 
estão associadas à metástase linfonodal, mas também outras clínicas e histopatológicas.

\section{Métodos}

Trata-se de estudo de coorte retrospectiva (coorte histórica) em que foram incluídas as pacientes admitidas no Departamento de Ginecologia do Centro de Tratamento e Pesquisa Hospital do Câncer A. C. Camargo com diagnóstico de carcinoma invasor do colo do útero no período de 1980 a 1999 e submetidas a tratamento cirúrgico radical. Os critérios estabelecidos para a inclusão das pacientes no estudo foram: I) comprovação histológica de carcinoma invasor; 2) estadiamento clínico IB e IIA segundo os critérios estabelecidos pela Federação Internacional de Ginecologia e Obstetrícia; 3) ausência de qualquer tratamento prévio à cirurgia, salvo conização para o diagnóstico; 4) realização de tratamento cirúrgico por histerectomia radical classes II ou III de Piver-Rutledge ${ }^{3}$ e linfonodectomia pélvica; 5) disponibilidade das lâminas contendo os cortes histopatológicos dos linfonodos para a revisão.

Os registros das pacientes foram identificados a partir dos bancos de dados disponíveis no Departamento de Ginecologia e no Serviço de Arquivos Médicos e Estatística sendo inicialmente selecionadas 466 pacientes. Após a revisão dos prontuários, foram excluídos 177 casos: 15 pacientes já tinham tratamento prévio à admissão, em dez casos as pacientes haviam sido submetidas a tratamento cirúrgico inadequado, I I 2 apresentavam estadiamento clínico diferente ao do interesse do estudo e em 27 casos o laudo anatomopatológico não revelou carcinoma invasor na peça operatória. Em 13 casos as lâminas com os cortes histopatológicos dos linfonodos não foram localizados. Após as exclusões, a população analisada foi composta por 289 mulheres, com a idade variando de 18 a 72 anos (média=44,4 anos; $d p=10,0$ anos; mediana $=44,0$ anos), em sua maioria brancas (75,8\%) ou pardas (16,6\%), casadas $(76,2 \%)$ e com baixo grau de instrução escolar (17,6\% eram analfabetas e 71,3\% apresentavam instrução até o ensino fundamental). A maioria das pacientes foi classificada clinicamente como IB $(90,7 \%)$ e as demais como IIA.

A partir da consulta dos prontuários, as informações foram coletadas em um formulário específico contendo dados sociodemográficos (idade ao diagnóstico, cor da pele), clínicos (estádio clínico da doença, taxa de hemoglobina pré-operatória e índice de massa corpórea) e algumas variáveis histopatológicas (tamanho do tumor e invasão dos ligamentos cervicais laterais e do corpo do útero). Para o estadiamento utilizou-se os critérios preconizados pela Federação Internacional de Ginecologia e Obstetrícia. A dosagem de hemoglobina foi realizada, em média, 26 dias antes da cirurgia $(\mathrm{d} p=\mid 8,9$ dias; mediana $=21$ dias), variando de 0 até 97 dias. 0 índice de massa corpórea foi calculado baseando-se no peso e altura das pacientes, os quais foram mensurados rotineiramente na véspera do procedimento cirúrgico. Em seis casos não se localizou no prontuário o valor da hemoglobina e em 60 não se pôde calcular o índice de massa corpórea por falta de informação sobre o peso e/ou altura.
Em 247 pacientes, as lâminas contendo os cortes histopatológicos dos colos de útero e dos linfonodos foram revistas por um único patologista conforme ficha padronizada através da observação em microscópio óptico Labophot$2\left(\right.$ Nikon $\left.^{\circledR}\right)$, sendo avaliadas as seguintes variáveis: tipo histológico, grau histológico, índice mitótico, embolização em capilares (linfáticos e/ou sangüíneos), invasão perineural, profundidade de invasão do tumor no estroma cervical (avaliada por microscopia), intensidade da reação inflamatória no colo do útero, necrose tumoral e status linfonodal. Destes casos, em dois os cortes do colo do útero não eram representativos o suficiente para a determinação do índice mitótico e da profundidade de invasão e em um caso para a avaliação de embolização capilar e de invasão perineural. Em 42 casos adicionais não foi possível rever os cortes histopatológicos do colo do útero em virtude da qualidade ruim das lâminas ou pela não localização das mesmas no arquivo do Departamento de Patologia. Todas as lâminas dos linfonodos foram revistas. O número final de casos com informação histopatológica ignorada mudou conforme a variável: tipo histológico (42), grau histológico (42), índice mitótico (44), tamanho do tumor (100), profundidade de invasão do tumor (44), embolização capilar (43), invasão perineural (43), invasão do ligamento cervical lateral (I68), invasão do corpo do útero (35), reação inflamatória no colo do útero (42) e necrose tumoral (42).

Todas as informações coletadas foram armazenadas em banco de dados informatizado e analisadas através do programa Statistical Package for Social Science - SPSS ${ }^{\circledR}$ (versão 13.0). A associação entre 0 status dos linfonodos pélvicos e as variáveis sociodemográficas, clínicas e histopatológicas foi avaliada inicialmente ou pelo teste de Qui-quadrado ou pelo teste exato de Fisher, dependendo dos valores esperados nas tabelas de contingência. As variáveis com nível descritivo de até 0,150 foram selecionadas para o modelo de regressão logística múltipla. Na análise multivariada, empregou-se a estratégia de modelagem do tipo stepwise selection e estipulou-se o nível de significância em 5\%. A estimativa de risco para a presença de metástases nos linfonodos pélvicos foi o odds ratio (OR) obtido da regressão logística múltipla.

Este trabalho faz parte de um estudo maior agregado à pósgraduação da Fundação Antônio Prudente e financiado pela Fundação de Amparo à Pesquisa do Estado de São Paulo. Foi previamento submetido e aprovado pela Comissão de Ética em Pesquisa do Centro de Tratamento e Pesquisa Hospital do Câncer A. C. Camargo (parecer $n^{\circ} 354 / 01$ ). Por se tratar de estudo retrospectivo, não houve necessidade de elaboração de termo de consentimento pós-informado.

\section{Resultados}

Observaram-se metástases nos linfonodos pélvicos através do estudo histopatológico em 65 casos (22,5\%). Nestas pacientes, o número de linfonodos acometidos variou de I a 20, com média de 2,5 ( $\mathrm{dp}=3,3$; mediana $=1,5)$.

$\mathrm{Na}$ análise univariada, das variáveis sociodemográficas e clínicas, somente a taxa de hemoglobina pré-operatória inferior a 10,0 
FreGNANI JHTG et aL.

$\mathrm{g} \%$ esteve associada às metástases linfonodais $(\mathrm{p}=0,005)($ Tabela 1). Das variáveis histopatológicas, mostraram associação com as metástases nos linfonodos o tipo histológico epidermóide versus adenocarcinoma $\quad(p=0,0 \mid 4), \quad$ carcinoma epidermóide queratinizante versus outros $(p=0,0 \mid 9)$, profundidade de invasão do tumor $(p=0,0||)$, presença de embolização capilar $(p=0,0 \mid 4)$ e presença de necrose tumoral $(p=0,020)$. Também foram selecionadas para a análise multivariada as seguintes variáveis: intensidade da reação inflamatória $(p=0,055)$, índice mitótico $(p=0,080)$ e tamanho do tumor $(p=0,08 \mathrm{I})$ (Tabela 2).

No modelo multivariado, as seguintes variáveis independentes mostraram-se associadas à presença de metástases nos linfonodos pélvicos: taxa de hemoglobina pré-operatória inferior a $10,0 \mathrm{~g} \%$, profundidade de invasão do tumor no colo do útero no terço médio ou no profundo, reação inflamatória ausente ou leve no colo do útero e tipo histológico compatível com carcinoma epidermóide queratinizante (Tabela 3).

\section{Discussão}

Este é um estudo de coorte retrospectiva realizado com pacientes portadoras de carcinoma do colo do útero de um centro de tratamento especializado em câncer ginecológico ao longo de quase duas décadas. Estudos retrospectivos estão sujeitos a críticas porque as informações são geralmente obtidas de prontuários médicos. Para melhorar a qualidade da informação, realizou-se revisão padronizada de dez variáveis histopatológicas. O fato desta revisão ter sido realizada por um único patologista reduziu o viés produzido pela variação entre observadores.

A freqüência de metástases nos linfonodos pélvicos neste estudo está de acordo com as taxas descritas na literatura para os estádios IB e IIA (I5,0 a 25,0\%). No presente estudo, quatro variáveis independentes foram associadas às metástases nos linfonodos pélvicos: a profundidade de invasão do tumor no colo do útero, a intensidade da reação inflamatória cervical, o tipo histológico e a taxa de hemoglobina pré-operatória. Das quatro, apenas a primeira é habitualmente citada na literatura como variável preditora de doença metastática nos linfonodos regionais no carcinoma do colo do útero.

A explicação para a maior freqüência de metástases linfonodais em tumores do colo do útero que invadem profundamente o estroma cervical está relacionada aos mecanismos de progressão e disseminação tumoral. Para que a neoplasia invada o estroma cervical, é preciso ocorrer a degradação da matriz extracelular, a qual é realizada por enzimas proteolíticas sintetizadas pelas células neoplásicas ${ }^{10-11}$. A digestão enzimática da matriz libera fragmentos que têm atividade pró-angiogênica e pró-linfangiogênica, formando novos capilares sangüíneos e linfáticos. $O$ aumento da densidade microvascular linfática é responsável pela disseminação das células neoplásicas para os linfonodos regionais ${ }^{12-15}$.

Neste estudo, as pacientes com anemia pré-operatória (taxa de hemoglobina inferior a 10,0 g\%) apresentaram metástases nos linfonodos pélvicos mais freqüentemente. Na literatura há diversos estudos demonstrando o valor prognóstico da anemia em pacien-

\begin{tabular}{|c|c|c|c|c|c|c|}
\hline \multirow[t]{2}{*}{ Variável } & \multirow[t]{2}{*}{ Categoria } & \multicolumn{2}{|c|}{$\begin{array}{c}\text { Sem } \\
\text { Metástase }\end{array}$} & \multicolumn{2}{|c|}{$\begin{array}{c}\text { Com } \\
\text { Metástase }\end{array}$} & \multirow[t]{2}{*}{$\mathrm{p}$} \\
\hline & & $\mathrm{n}(*)$ & (\%) & $\mathrm{n}(*)$ & (\%) & \\
\hline Idade & $\begin{array}{l}\text { Até } 35 \text { anos } \\
\text { Mais de } 35 \text { anos }\end{array}$ & $\begin{array}{l}47 \\
194\end{array}$ & $\begin{array}{l}(81,0) \\
(84,0)\end{array}$ & $\begin{array}{l}11 \\
37\end{array}$ & $\begin{array}{l}(19,0) \\
(16,0)\end{array}$ & 0,590 \\
\hline Corda pele & $\begin{array}{l}\text { Branca } \\
\text { Não branca }\end{array}$ & $\begin{array}{l}179 \\
62\end{array}$ & $\begin{array}{l}(81,7) \\
(88,6)\end{array}$ & $\begin{array}{l}40 \\
8\end{array}$ & $\begin{array}{l}(18,3) \\
(11,4)\end{array}$ & 0,181 \\
\hline Estádio clínico & $\begin{array}{l}\mathbb{B} \\
\| A\end{array}$ & $\begin{array}{l}217 \\
24\end{array}$ & $\begin{array}{l}(82,8) \\
(88,9)\end{array}$ & $\begin{array}{c}45 \\
3\end{array}$ & $\begin{array}{l}(17,2) \\
(11,1)\end{array}$ & 0,589 \\
\hline $\begin{array}{l}\text { Taxade } \\
\text { hemoglobina } \\
\text { pré-operatória (g\%) }\end{array}$ & $\begin{array}{l}<10,0 \\
10,0 \text { ou mais }\end{array}$ & $\begin{array}{l}11 \\
226\end{array}$ & $\begin{array}{l}(57,9) \\
(85,6)\end{array}$ & $\begin{array}{c}8 \\
38\end{array}$ & $\begin{array}{l}(42,1) \\
(14,4)\end{array}$ & 0,005 \\
\hline $\begin{array}{l}\text { Índice de massa } \\
\text { corpórea }\left(\mathrm{kg} / \mathrm{m}^{2}\right)\end{array}$ & $\begin{array}{l}<21 \\
21-25 \\
>25\end{array}$ & $\begin{array}{l}34 \\
76 \\
78\end{array}$ & $\begin{array}{l}(81,0) \\
(82,6) \\
(82,1)\end{array}$ & $\begin{array}{l}8 \\
16 \\
17\end{array}$ & $\begin{array}{l}(19,0) \\
(17,4) \\
(17,9)\end{array}$ & 0,973 \\
\hline
\end{tabular}

* Casos com valores ignorados foram excluídos da análise: taxa de hemoglobina préoperatória (6) e índice de massa corpórea (60).

tes portadoras de carcinoma do colo do útero e tratadas com radioterapia ${ }^{16-17}$. No entanto, não se conhece estudo prévio que tenha verificado a anemia como possível fator preditor de metástases linfonodais no carcinoma do colo do útero.

Há fortes evidências de que a anemia seja capaz de determinar baixa oxigenação tumoral e, com isso, induzir a angiogênese através do aumento da expressão de fatores de crescimento endotelial, como o VEGF (vascular endothelial growth factor) ${ }^{18}$. 0 aumento da densidade microvascular no tumor facilitaria a disseminação tumoral pelos vasos sangüíneos. É preciso recordar que, embora menos freqüentemente, as células neoplásicas podem chegar aos linfonodos pela via hematogênica através de anastomoses dos sistemas linfático e sangüíneo ${ }^{10,13}$. Apesar de se acreditar que a expressão do principal fator pró-linfangiogênico (VEGF-C) não seja induzida pela hipoxia tumoral'2, é provável que a linfangiogênese também seja estimulada pela anemia, uma vez que a angiogênese e a linfangiogênese são eventos freqüentemente coexistentes $^{10}$. O fato é que, nas pacientes portadoras de anemia pré-operatória, o risco de metástases nos linfonodos pélvicos foi cerca de quatro vezes maior quando a variável foi ajustada pelas outras. Este resultado não pode ser atribuído ao acaso e merece investigações futuras mais detalhadas, buscando elucidar uma possível associação entre a hipoxia tumoral e os mecanismos indutores da linfangiogênese.

Observou-se que o carcinoma epidermóide queratinizante apresentou metástases nos linfonodos regionais mais freqüentemente que os demais tipos histológicos. Classicamente considerase como bem diferenciado o carcinoma epidermóide produtor de queratina $^{19}$. Parece estranho que um tumor com esta diferenciação 
Tabela 2-Número e porcentagem de pacientes segundo a presença de metástases nos linfonodos pélvicos e as variáveis histopatológicas (Hospital do Câncer A. C. Camargo, 1980-1999)

\begin{tabular}{|c|c|c|c|c|c|c|}
\hline Variável & Categoria & Sen & tase & Com & tase & $\mathrm{p}$ \\
\hline & & $\mathrm{n}(*)$ & (\%) & $\mathrm{n}(*)$ & $(\%)$ & \\
\hline Tipo histológico & $\begin{array}{l}\text { Epidermóide queratinizante } \\
\text { Outros tipos }\end{array}$ & $\begin{array}{l}30 \\
173\end{array}$ & $\begin{array}{l}(69,8) \\
(84,8)\end{array}$ & $\begin{array}{l}13 \\
31\end{array}$ & $\begin{array}{l}(30,2) \\
(15,2)\end{array}$ & 0,019 \\
\hline & $\begin{array}{l}\text { Epidermóide } \\
\text { Adenocarcinoma }\end{array}$ & $\begin{array}{l}164 \\
34\end{array}$ & $\begin{array}{l}(80,0) \\
(97,1)\end{array}$ & $\begin{array}{c}41 \\
1\end{array}$ & $\begin{array}{l}(20,0) \\
(2,9)\end{array}$ & 0,014 \\
\hline Grau histológico & $\begin{array}{l}1 \\
2 \\
3 \text { ou } 4\end{array}$ & $\begin{array}{l}39 \\
97 \\
67\end{array}$ & $\begin{array}{l}(88,6) \\
(78,2) \\
(84,8)\end{array}$ & $\begin{array}{c}5 \\
27 \\
12\end{array}$ & $\begin{array}{l}(11,4) \\
(21,8) \\
(15,2)\end{array}$ & 0,229 \\
\hline $\begin{array}{l}\text { Índice mitótico } \\
\text { (em } 10 \text { campos de } \\
\text { grande aumento) }\end{array}$ & $\begin{array}{l}\text { Até } 10 \\
\text { II a } 20 \\
\text { Mais de } 20\end{array}$ & $\begin{array}{l}55 \\
59 \\
87\end{array}$ & $\begin{array}{l}(91,7) \\
(79,7) \\
(78,4)\end{array}$ & $\begin{array}{l}5 \\
15 \\
24\end{array}$ & $\begin{array}{l}(8,3) \\
(20,3) \\
(21,6)\end{array}$ & 0,080 \\
\hline Tamanho do tumor (cm) & $\begin{array}{l}\text { Até2 } \\
2,1 \text { a } 4,0 \\
\text { Mais do que } 4\end{array}$ & $\begin{array}{l}47 \\
87 \\
24\end{array}$ & $\begin{array}{l}(81,0) \\
(88,8) \\
(72,2)\end{array}$ & $\begin{array}{l}11 \\
11 \\
9\end{array}$ & $\begin{array}{l}(19,0) \\
(11,1) \\
(27,3)\end{array}$ & 0,081 \\
\hline Profundidade de invasão do tumor & $\begin{array}{l}\text { Terço superficial } \\
\text { Terço médio } \\
\text { Terço profundo }\end{array}$ & $\begin{array}{l}58 \\
79 \\
64\end{array}$ & $\begin{array}{l}93,5 \\
81,4 \\
74,4\end{array}$ & $\begin{array}{l}4 \\
18 \\
22\end{array}$ & $\begin{array}{l}6,5 \\
18,5 \\
25,6\end{array}$ & 0,011 \\
\hline Embolização capilar & $\begin{array}{l}\text { Não } \\
\text { Sim }\end{array}$ & $\begin{array}{l}110 \\
92\end{array}$ & $\begin{array}{l}(88,0) \\
(76,0)\end{array}$ & $\begin{array}{l}15 \\
29\end{array}$ & $\begin{array}{l}(12,0) \\
(24,0)\end{array}$ & 0,014 \\
\hline Invasão perineural & $\begin{array}{l}\text { Não } \\
\text { Sim }\end{array}$ & $\begin{array}{l}176 \\
26\end{array}$ & $\begin{array}{l}(81,5) \\
(86,7)\end{array}$ & $\begin{array}{c}40 \\
4\end{array}$ & $\begin{array}{l}(18,5) \\
(13,3)\end{array}$ & 0,487 \\
\hline Invasão do ligamento cervical lateral & $\begin{array}{l}\text { Não } \\
\text { Sim }\end{array}$ & $\begin{array}{c}94 \\
5\end{array}$ & $\begin{array}{l}(82,5) \\
(71,4)\end{array}$ & $\begin{array}{l}20 \\
2\end{array}$ & $\begin{array}{l}(17,5) \\
(28,6)\end{array}$ & 0,610 \\
\hline Invasão do corpo do útero & $\begin{array}{l}\text { Não } \\
\text { Sim }\end{array}$ & $\begin{array}{l}184 \\
30\end{array}$ & $\begin{array}{l}(83,6) \\
(88,2)\end{array}$ & $\begin{array}{c}36 \\
4\end{array}$ & $\begin{array}{l}(16,4) \\
(11,8)\end{array}$ & 0,493 \\
\hline Reação inflamatória no colo do útero & $\begin{array}{l}\text { Ausente ou leve } \\
\text { Moderada ou intensa }\end{array}$ & $\begin{array}{l}111 \\
92\end{array}$ & $\begin{array}{l}(78,2) \\
(87,6)\end{array}$ & $\begin{array}{l}31 \\
13\end{array}$ & $\begin{array}{l}(21,8) \\
(12,4)\end{array}$ & 0,055 \\
\hline Necrose tumoral & $\begin{array}{l}\text { Não } \\
\text { Sim }\end{array}$ & $\begin{array}{l}79 \\
124\end{array}$ & $\begin{array}{l}(89,8) \\
(78,0)\end{array}$ & $\begin{array}{c}9 \\
35\end{array}$ & $\begin{array}{l}(10,2) \\
(22,0)\end{array}$ & 0,020 \\
\hline
\end{tabular}

* Casos com valores ignorados foram excluídos da análise: tipo histológico (42), grau histológico (42), índice mitótico (44), tamanho do tumor (100), profundidade de invasão do tumor (44), embolização capilar (43), invasão perineural (43), invasão do ligamento cervical lateral (168), invasão do corpo do útero (35), reação inflamatória no colo do útero (42) e necrose tumoral (42).

tenha um potencial metastático maior do que os demais. Vale ressaltar que, do ponto de vista histológico, o epitélio de revestimento do colo do útero é estratificado pavimentoso nãoqueratinizado ${ }^{20}$. Assim, a produção de queratina não é condição habitual e normal para as células epiteliais do colo do útero, embora possam fazê-lo em condições de doença. A síntese de queratina indicaria uma mudança do perfil genético das células epiteliais cervicais. É razoável acreditar que esta mudança não abrange somente os genes relacionados à expressão da queratina, mas, talvez se estenda a outros. É provável que alguns dos genes associados aos mecanismos de progressão e disseminação tumoral também sofram mudança em suas expressões. A observação de queratina em um carcinoma epidermóide do colo do útero representaria sinal histopatológico indireto de mudança do perfil genético das células, sendo estas portadoras de maior potencial metastático. Esta é uma hipótese meramente especulativa e precisa ser confirmada em outros estudos.

Os trabalhos já publicados acerca da queratinização no carcinoma do colo do útero não analisaram esta variável como possível fator preditor de metástases nos linfonodos regionais, mas procuraram verificar o seu valor prognóstico ${ }^{21-22}$. Ao que parece, esta é a primeira vez que se descreve a queratinização como fator de risco no aparecimento de metástases linfonodais pélvicas no carcinoma do colo do útero.

Quando em resposta ao tumor a reação inflamatória no colo do útero foi débil (ausente ou leve), isto determinou maior risco de 


\begin{tabular}{|c|c|c|c|c|}
\hline Variável & Categoria & $\begin{array}{c}\text { OR } \\
\text { (bruta) }\end{array}$ & $\begin{array}{c}\text { OR } \\
\text { (ajustada) }\end{array}$ & $\begin{array}{c}\text { IC95\% } \\
\text { (ORajustada) }\end{array}$ \\
\hline $\begin{array}{l}\text { Taxa de } \\
\text { hemoglobina } \\
\text { pré-operatória }\end{array}$ & $\begin{array}{l}10,0 \mathrm{~g} \% \text { ou mais } \\
\text { Inferior a } 10,0 \mathrm{~g} \%\end{array}$ & $\begin{array}{l}1,0 \\
4,3\end{array}$ & $\begin{array}{l}1,0 \\
3,6\end{array}$ & $\begin{array}{c}\text { Ref. } \\
1,2-10,7\end{array}$ \\
\hline $\begin{array}{l}\text { pre-operatorla } \\
\text { Profundidade de } \\
\text { invasão do tumor }\end{array}$ & $\begin{array}{l}\text { Terço superficial } \\
\text { Terço médio } \\
\text { Terço profundo }\end{array}$ & $\begin{array}{l}1,0 \\
3,3 \\
5,0\end{array}$ & $\begin{array}{l}1,0 \\
3,3 \\
5,4\end{array}$ & $\begin{array}{c}\text { Ref. } \\
1,1-10,7 \\
1,7-17,3\end{array}$ \\
\hline $\begin{array}{l}\text { Reação inflamatória } \\
\text { no colo do útero }\end{array}$ & $\begin{array}{l}\text { Moderada ou intensa } \\
\text { Ausente ou leve }\end{array}$ & $\begin{array}{l}1,0 \\
2,0\end{array}$ & $\begin{array}{l}1,0 \\
2,4\end{array}$ & $\begin{array}{c}\text { Ref. } \\
|,|-5,2\end{array}$ \\
\hline Tipo histológico & $\begin{array}{l}\text { Outros tipos } \\
\text { Epidermóide queratinizante }\end{array}$ & $\begin{array}{l}1,0 \\
2,4\end{array}$ & $\begin{array}{l}1,0 \\
3,3\end{array}$ & $\begin{array}{c}\text { Ref. } \\
1,4-7,6\end{array}$ \\
\hline $\begin{array}{l}\text { OR } \\
\text { IC95\% } \\
\text { Ref. }\end{array}$ & de confiança de $95 \%$ & & & \\
\hline
\end{tabular}

metástases nos linfonodos. Este fato deve ser compreendido lembrando-se que o sistema imune desempenha importante função no controle supressor das neoplasias. A falha no sistema de vigilância imunitária, sobretudo da imunidade celular, tem sido implicada não somente na carcinogênese, mas, também, na progressão e disseminação das células neoplásicas ${ }^{23-25}$. Sabe-se que as células tumorais conseguem escapar dos mecanismos de vigilância imunitária, promovendo a depressão da imunidade celular. Há diversos mediadores envolvidos na depressão imunitária que são produzidos pelas células neoplásicas, destacando-se os seguintes: TGF-b (transforming growth factor-beta) interleucina IL-4, interleucina IL-6, interleucina IL-10, MCP-I (macrophage chemotactic protein-I), MDF (macrophage desativating factor), SER (suppressive E-receptor), IAP (immunosuppressive acidic protein), prostaglandina PGE2, proteína pI5E, metaloproteinases, entre outros ${ }^{26-27}$. Não é de se surpreender, portanto, que a ausência de resposta inflamatória efetiva ao carcinoma do colo do útero esteja associada à maior freqüência de metástases nos linfonodos pélvicos. Os resultados aqui apresentados estão em concordância com os de Bethwaite et al. que observaram significativa associação entre a baixa densidade de linfócitos $T$ no tumor e a presença de metástases nos linfonodos em 73 pacientes com carcinoma do colo do útero (estádio IB) ${ }^{28}$. Mais recentemente, em um estudo com 59 mulheres portadoras de carcinoma do colo do útero, Piersma et al. reportaram menores taxas de metástase nos linfonodos de pacientes cujos colos estavam densamente infiltrados por linfócitos CD8 $+{ }^{29}$.

\section{Conclusão}

Este estudo observou que a presença de metástase linfonodal pélvica no carcinoma do colo do útero estádios IB e IIA esteve associada à invasão do terço médio e profundo do colo do útero, à anemia pré-operatória (taxa de hemoglobina inferior à 10,0g\%), à reação inflamatória no colo do útero ausente ou de intensidade leve e ao tipo histológico compatível com carcinoma epidermóide queratinizante. Destas, somente a primeira é classicamente citada na literatura como fator de risco para metástase nos linfonodos. Fica aqui registrada a necessidade de maior atenção aos outros três fatores de risco para metástase nos linfonodos. Pacientes consideradas livres de doença nos linfonodos pélvicos, mas detentoras de algum dos fatores de risco supracitados talvez devessem ter os seus linfonodos revistos em busca de possível metástase não identificada no exame histopatológico convencional.

\section{Conflito de interesse: não há.}

\section{SUMMARY \\ UNUSUAL RISK FACTORS FOR LYMPH NODE METASTASIS IN CANCER OF THE UTERINE CERVIX}

OBIECTIVE. To investigate the variables associated with pelvic lymph node metastasis in patients with carcinoma of the uterine cervix at stages IB and IIA.

Methods. The study was carried out with 289 patients with invasive carcinoma of the uterine cervix (IB and IIA) who underwent radical surgery, admitted to the Gynecology Department of the Treatment and Research Center of the Hospital do Câncer A. C. Camargo, between 1980 and 1999. Data were collected from patient's records and from review of the histological sections from the uterine cervices and the lymph nodes, including sociodemographic data (age at diagnosis, skin color), clinical data (disease stage, preoperative hemoglobin level, body mass index) and histopathological data (histological type, histological grade, blood and/or lymphatic capillary embolization, perineural invasion, depth of tumor invasion into cervical stroma, intensity of inflammatory reaction in the uterine cervix, tumor necrosis, tumor size, invasion of the lateral cervical ligaments and uterine body, and lymph node status). Associations between lymph node metastasis and the different variables were evaluated by means of the chisquare test, Fisher's exact test and multiple logistic regression.

RESULTS. Pelvic lymph node metastasis was found in 65 patients (22.5\%). The following risk factors for lymph node metastasis were identified by multivariate analysis: hemoglobin level $<10.0$ $g \%(O R=3.6 ; 95 \% \mathrm{Cl}: 1.2-10.7)$, tumor invasion of the middle third $(O R=3.3 ; 95 \% \mathrm{Cl}: 1.1-10.7)$ and deep third of the uterine cervix $(O R=5.4 ; 95 \%$ Cl: 1.7-17.3), absent or slight inflammatory reaction in the uterine cervix $(O R=2.4 ; 95 \% \mathrm{Cl}$ : I.1-5.2) and keratinizing squamous cell carcinoma $(O R=3.3$; 95\% Cl: 1.4-7.6).

Conclusions. This study identified four risk factors for pelvic lymph node metastasis in patients with carcinoma of the uterine cervix at stages IB and IIA. Of these, three are not usually described in literature as predictive variables for lymph node metastasis (preoperative anemia, absent or slight inflammatory reaction and keratinizing of squamous cell carcinoma). [Rev Assoc Med Bras 2007; 53(4): 338-43]

KEY WORDS: Uterine cervical neoplasms. Lymphatic metastases. Lymph nodes. Anemia. Inflammation. 


\section{REFERÊNCIAS}

I. Parkin DM, Bray FI, Devesa SS. Cancer burden in the year 2000: the global picture. Eur J Cancer. 2001;37(Suppl 8):S4-66.

2. Benedet JL, Odicino F, Maisonneuve P, Beller U, Creasman WT, Heintz AP, et al. Carcinoma of the cervix uteri. Int J Gynaecol Obstet. 2003;83(Suppl I):4 I-78.

3. Piver MS, Rutledge F, Smith JP. Five classes of extended hysterectomy for women with cervical cancer. Obstet Gynecol. 1974:44:265-72.

4. Kesic V. Management of cervical cancer. Eur J Surg Oncol. 2006:32:832-7

5. Wang CJ, Lai $\mathrm{CH}$, Huang $\mathrm{HJ}$, Hong JH, Chou HH, Huang KG, et al. Recurrent cervical carcinoma after primary radical surgery. Am J Obstet Gynecol. 1999;181:518-24.

6. Hart K, Han I, Deppe G, Malviya V, Malone J Jr, Christensen C, et al. Postoperative radiation for cervical cancer with pathologic risk factors. Int J Radiat Oncol Biol Phys. 1997;37:833-8.

7. Fregnani JH, Latorre MR, Novik PR, Lopes A, Soares FA. Assessment of pelvic lymph node micrometastatic disease in stages IB and IIA of carcinoma of the uterine cervix. Int J Gynecol Cancer. 2006; |6: I 18894.

8. Singh N, Arif S. Histopathologic parameters of prognosis in cervical cancer-a review. Int J Gynecol Cancer. 2004; | 4:74I-50.

9. Silva-Filho AL, Reis FM, Traiman P, Pedrosa MS, Miranda D, Triginelli SA. Clinicopathological features influencing pelvic lymph node metastasis and vaginal and parametrial involvement in patients with carcinoma of the cervix. Gynecol Obstet Invest. 2005;59:92-6.

10. Nathanson SD. Insights into the mechanisms of lymph node metastasis. Cancer. 2003;98:4I3-23.

I I. Horn LC, Richter CE, Hentschel B, Schutz A, Pilch H, Leo C, et al. Juxtatumoral desmoplastic stromal reaction is associated with high tumor cell dissociation in squamous cell carcinomas of the uterine cervix. Ann Diagn Pathol. 2006;10:253-6.

12. Stacker SA, Baldwin ME, Achen MG. The role of tumor lymphangiogenesis in metastatic spread. FASEB J. 2002;16:922-34.

1 3. He Y, Karpanen T, Alitalo K. Role of lymphangiogenic factors in tumor metastasis. Biochim Biophys Acta. 2004;1654:3-12.

I 4. Gao P, Zhou GY, Yin G, Liu Y, Liu ZY, Zhang J, et al. Lymphatic vessel density as a prognostic indicator for patients with stage I cervical carcinoma. Hum Pathol. 2006;37:719-25.

15. Gombos Z, Xu X, Chu CS, Zhang PJ, Acs G. Peritumoral lymphatic vessel density and vascular endothelial growth factor $C$ expression in early-stage squamous cell carcinoma of the uterine cervix. Clin Cancer Res.2005; I I:8364-7 I.

16. Fyles AW, Milosevic M, Hedley D, Pintilie M, Levin W, Manchul L, et al. Tumor hypoxia has independent predictor impact only in patients with node-negative cervix cancer. J Clin Oncol. 2002;20:680-7.

17. Kummel S, Thomas A, Jeschke S, Hauschild M, Sehoule J, Lichtenegger $W$, et al. Postoperative therapy modalities for cervical carcinoma. Anticancer Res. 2006;26:1707-13.
18. Dunst J, Becker A, Lautenschlager C. Anemia and elevated systemic levels of vascular endothelial growth factor (VEGF). Strahlenther Onkol. 2002; | 78:436-41.

19. Torres W. Câncer do colo uterino: aspectos anatomopatológicos. In: Figueiredo E, editor. Ginecologia Oncológica. Rio de Janeiro: Revinter; 2004.p.26-31.

20. Fawcett DW. Female reproductive system. In: Fawcett DW, editor. Bloom and Fawcett, a textbook of histology. 12 ${ }^{\text {th }}$ ed. New York: Chapman \& Hall; 1994.p.816-60.

21. Zaino RJ, Ward S, Delgado G, Bundy B, Gore H, Fetter G, et al. Histopathologic predictors of the behavior of surgically treated stage IB squamous cell carcinoma of the cervix. A Gynecologic Oncology Group study. Cancer. 1992;69:1750-8.

22. Graflund M, Sorbe B, Hussein A, Bryne M, Karlsson M. The prognostic value of histopathologic grading parameters and microvessel density in patients with early squamous cell carcinoma of the uterine cervix. Int J Gynecol Cancer. 2002; 12:32-41.

23. Seliger B. Strategies of tumor immune evasion. BioDrugs. 2005; 1 9:347-54.

24. Yu P, Fu YX. Tumor-infiltrating $T$ lymphocytes: friends or foes? Lab Invest. 2006;86:231-45.

25. Sheu BC, Chiou SH, Lin HH, Chow SN, Huang SC, Ho HN, et al. Upregulation of inhibitory natural killer receptors CD94/NKG2A with suppressed intracellular perforin expression of tumor-infiltrating CD8+ $T$ lymphocytes in human cervical carcinoma. Cancer Res. 2005;65:2921-9.

26. Botti C, Seregni E, Ferrari L, Martinetti A, Bombardieri E. Immunosuppressive factors: role in cancer development and progression. Int J Biol Markers. 1998; 13:51-69.

27. Elgert KD, Alleva DG, Mullins DW. Tumor-induced immune dysfunction: the macrophage connection. J Leukoc Biol. 1998;64:27590

28. Bethwaite PB, Holloway LJ, Thornton A, Delahunt B. Infiltration by immunocompetent cells in early stage invasive carcinoma of the uterine cervix: a prognostic study. Pathology. 1996;28:321-7.

29. Piersma SJ, Jordanova ES, Van Poelgeest MI, Kwappenberg KM, Van der Hulst JM, Dryfhout JW, et al. High number of intraepithelial CD8+ tumor-infiltrating lymphocytes is associated with the absence of lymph node metastases in patients with large early-stage cervical cancer. Cancer Res. 2007;67:354-61.

Artigo recebido: 30/1 1/06 Aceito para publicação: 21/02/07 